\title{
Leaf senescence induced by EGY1 defection was partially restored by glucose in Arabidopsis thaliana
}

\author{
Cuiyun Chen", Jin Wang and Xin Zhao
}

\begin{abstract}
Background: Ethylene-dependent gravitropism-deficient and yellow-green 1 (EGY1) protein is required for chloroplast development and photosynthesis conduction. The egy 1 deletion mutants have a yellow-green phenotype and reduced granal thylakoids. Furthermore, the yellow-green phenotype of egy 1 mutants is more obvious than that of wild-type (WT) plants with increasing leaf age, suggesting an early senescence in the egy 1 mutants. However, the relationship between EGY1 functions and leaf senescence still remains poorly understood.

Results: We observed that egy 1 mutant leaves were more yellow than those of WT (the same age) in Arabidopsis thaliana. In accompany with this phenotype, leaf survival, chlorophyll content, Fv/Fm and soluble protein content decreased, and ion leakage increased significantly in egy 1 mutants compared to WT plants. At molecular level, the expressions of senescence-associated genes increased, and photosynthesis genes decreased significantly in the mutants compared to those in WT plants. Furthermore, after darkness treatment, the yellow-green phenotype of egy 1 mutants was more obvious than that of WT. These results indicate that the loss-of-function of egy 1 gene induces leaf senescence in A. thaliana. In addition, our results showed that the yellow-green phenotype, chlorophyll content and ion leakage of egy 1 mutants was partially restored after exogenously applied glucose for 5 weeks. At the same time, the expression of hexokinase 1 (HXK1) and/or senescence-associated gene 12 (SAG12) in egy 1 mutants growing on $2 \%$ glucose was lower than that in egy 1 mutants without glucose.
\end{abstract}

Conclusion: EGY1-defection induced leaf senescence and this senescence was partially restored by glucose in $A$. thaliana.

Keywords: egy1, Leaf senescence, Glucose, Senescence-associated genes

\section{Background}

The leaf is a specialized photosynthetic organ of plants, serving as the major site of producing energy and nutrients. Its development stages include initiation, growth, differentiation, maturation and senescence. Leaf senescence, the last stage of leaf development, plays a key role in plant survival and/or death. During leaf senescence, plant cells undergo orderly changes in structure, metabolism and gene expression (Buchanan-Wollaston et al. 2003; Guiboileau et al. 2010). Among these, the

\footnotetext{
${ }^{*}$ Correspondence: chency@|zb.ac.cn

Stress Physiology and Ecology Laboratory, Cold and Arid Regions

Environment and Engineering Research Institute, Chinese Academy of Sciences, Lanzhou 730000, China
}

degradation processes of chlorophyll, proteins and lipids have been largely investigated (Hortensteiner and Feller 2002; Wiedemuth et al. 2005). Chlorophyll degradation typically starts at the leaf margins and progresses to the interior of the leaf blade (Kakani et al. 2004). Protein degradation is the most significant breakdown process that takes place during senescence (Buchanan-Wollaston et al. 2003). Other organelles, such as the liposome, also undergo biochemical changes as senescence proceeds (Hung and Kao 1998).

Leaf senescence is usually triggered by internal agedependent factors, which include the expression changes of senescence-associated genes (SAGs), and the mutants of leaf death inducing genes, jasmonic acid-associated

\section{黛 Springer}


genes and other key genes (Castillo and Leon 2008; Guo et al. 2004). However, some external factors such as the alterations of nutrient, light and other environmental factors also initiate leaf senescence (Guiboileau et al. 2010; Ono et al. 2001). Of course, the defect of a gene associated with producing a nutrient (such as sugar) has the same effects as the external factors mentioned above (Loreti et al. 2008). Sugar signaling pathways are important regulation mechanisms for leaf senescence in plants (van Doorn 2008), which consists of glucose, sucrose, trehalose and other hexokinase-independent sugar signaling pathways (Smeekens et al. 2009; Xiao et al. 2000). Among these, the glucose signaling pathway is considered as one of the most important mechanism responsible for senescence and has been extensively studied (Balasubramanian et al. 2008). And hexokinase 1 (HXK1) acts as the direct glucose sensor mediating multiple functions in the glucose repression and glucose promotion of transcription and growth (Cho et al. 2006).

Nuclear mutations that cause albino or pale green phenotypes because of reduced levels of chlorophyll in the chloroplasts have been found frequently in higher plants (Bellaoui and Gruissem 2004; Vinti et al. 2005). These mutants usually have normal leaf anatomies, but typically show defects in chloroplast ultrastructure and composition (Aluru et al. 2007). The mutants of egy1 (which coding for a protein named ethylene-dependent gravitropism-deficient and yellow-green 1, EGY1) are such plants of Arabidopsis thaliana because EGY1 protein is required for chloroplast development and photosynthesis conduction (Chen et al. 2005; Guo et al. 2008), and the yellow-green phenotype of egy1 mutants is more obvious than that of wild-type (WT) plants with increasing leaf age. Although the photosynthesis characteristics of egy 1 mutants have been studied extensively, the relation between egy1 mutation and leaf senescence is completely unknown. Since there was little attention given to color mutants and leaf senescence (Yoshida et al. 2002), we aim to explore the effects of EGY1-defection on leaf senescence and to further elaborate its potential mechanism in this paper.

\section{Methods}

\section{Plant materials and growth conditions}

The T-DNA insertion line SALK-134931 was obtained from the Arabidopsis Biological Resource Center (ABRC, Ohio State University), and plants homozygous for the egy1 mutation (also known as egy1-2 in Chen et al. 2005) were used for further analysis. Wild-type and the mutant plants were grown under 10-h-light/14-h-dark cycle with a photon flux density of $120 \mu \mathrm{mol} \mathrm{m}{ }^{-2} \mathrm{~s}^{-1}$ at $22{ }^{\circ} \mathrm{C}$. To ensure synchronized germination, the seeds were sown and then maintained in darkness for $48 \mathrm{~h}$ at $4{ }^{\circ} \mathrm{C}$.
For dark treatment, 3-week-old seedlings of WT and egy1 mutant plants were placed in complete darkness for 3 days at $22^{\circ} \mathrm{C}$.

\section{Complementation of the egy 1 mutants}

For complementation of the egy1 mutants, cDNA containing the EGY1 coding region was amplified by PCR with the sense primer 5'-GGATCCAATGGGGACTCTC AC-CAG-3' and antisense primer 5'-CGAGCTCTCACT AGTGTACATACATGGC-3'. The PCR product was cleaved with BamHI and SacI and cloned into the plant expression vector pSN1301 under the control of the cauliflower mosaic virus $35 \mathrm{~S}$ promoter. The construct was transformed into Agrobacterium tumefaciens strain C58 and introduced into egy1 plants by the floral dip method (Clough and Bent 1998). Transformed plants were selected on MS medium (Murashige and Skoog basal liquid medium; Sigma-Aldrich) containing $50 \mathrm{mg} \mathrm{mL}$ hygromycin. The success of the complementation procedure was confirmed by PCR analysis and chlorophyll contents detection on the resulting plants.

\section{Measurement of leaf survival, chlorophyll content, photochemical efficiency, soluble protein and ion leakage}

All these parameters were measured with the fifth rosette leaves, which were harvested at specified days counted from the day of leaf emergence. Leaf survival was determined by the mortality curves, which was the percentage of leaves that didn't show full yellow (full yellow leaf was considered as dead) versus all leaves. Chlorophyll contents were quantified as described by Sims and Gamon (2002).

The photochemical efficiency of PSII was deduced from chlorophyll fluorescence parameters measured using a portable plant efficiency analyzer (Hansatech Instruments, Morfolk, England). The ratio of maximum variable fluorescence to maximum yield of fluorescence $(F v / F m)$ was used as a measure of the photochemical efficiency of PSII.

Protein was extracted in $10 \mathrm{mM}$ Hepes-KOH $(\mathrm{pH}$ 8.0), $10 \mathrm{mM} \mathrm{MgCl} 2,330 \mathrm{mM}$ sorbitol, $2 \mathrm{mM}$ PMSF and concentration was determined with a protein assay kit (Bio-Rad, CA, USA) using bovine serum albumin as a standard. Membrane ion leakage was determined by measuring electrolytes leaked using a digital conductivity.

\section{Nucleic acid preparation and analysis}

Total RNA was extracted from $100 \mathrm{mg}$ of fresh tissues using TRIzol reagent (Invitrogen). For the determination of the gene's expression, RT-PCR was performed using the following primers: sense (5'-TCGCTTTTGCCGCTGCC GTTAACATTAG-3') and antisense (5'-AAAGCTAA CACGAGCACCACCGCGAGG-3'). To ensure equal 
amount of RNA in each sample, RT-PCR analysis of actin cDNA was performed using the following primers: sense (5'-AACTGGGATGATATGGAGAA- $3^{\prime}$ ) and antisense (5'-CCTCCAATCCAGACACTGTA-3').

Northern blot analyses were performed essentially as described previously (Sambrook and Russell 2001). The fifth leaves were harvested from WT and egy1 plants at 26, 33 and 40 days after germination, and RNAs were separated on a $1.0 \%$ agarose/formaldehyde gel, then transferred onto Nylon membranes (Amersham Pharmacia Biotech). The membranes were probed with ${ }^{32} \mathrm{P}$-labelled cDNA probes specific for senescence-associated gene 12 (SAG12), SAG24, endo-xyloglucan transferase/xyloglucan endo-1,4-beta-D-glucanase (SEN4), chlorophyll a/bbinding protein $(C A B)$, and the rubisco small subunit gene $(R B C S)$. Ethidium bromide staining was used as a loading control. Following high-stringency hybridization and washing, all the blots were exposed to X-ray film.

\section{Exogenous glucose treatment}

Arabidopsis thaliana seeds were sown on half-strength MS plates containing various concentrations of glucose, and plates were placed at $4{ }^{\circ} \mathrm{C}$ for 2 days for vernalization. They were germinated and maintained at $22{ }^{\circ} \mathrm{C}$ in darkness for 6 days and illuminated at $22^{\circ} \mathrm{C}$ for $12 \mathrm{~h}$. The hypocotyl lengths of seedlings were measured.

At the same time, WT and egy1 plants were grown on $2 \%$ glucose + MS medium in a 500 cubic $\mathrm{cm}$ glass jar for 5 weeks, and then the phenotype were observed, the chlorophyll contents, ion leakage were determined, and the expressions of hexokinase 1 (HXK1) and senescenceassociated gene 12 (SAG12) were quantified.

\section{Quantitative PCR Analysis}

Primer pairs for the quantitative PCR were: $5^{\prime}$-GCAGA CTTCTCTGTCCTCTGG-TAG-3' (forward) and 5'-TC CAACAACATCTTGTCCAACTGC-3' (reverse) for HXK1; 5'-AAGGAGGAAAACAATCGCTAC-3' (forward) and 5'-GCAAACTGA-TTTACCGCAAG-3' (reverse) for SAG12; 5'-CGTACAACCGGTATTGTGC T-GG-3' (forward) and 5'-CTCTCTCTGTAAGGATCT TCATG-3' (reverse) for actin. The quantitative PCR was performed with a Mx3000P Real-Time PCR System (Stratagene, Agilent, USA) using SYBR Green SuperMix (Takara) with the following conditions: $30 \mathrm{~s}$ at $95^{\circ} \mathrm{C}$; 40 cycles of $10 \mathrm{~s}$ at $95{ }^{\circ} \mathrm{C}, 10 \mathrm{~s}$ at $55^{\circ} \mathrm{C}$ and $12 \mathrm{~s}$ at $72{ }^{\circ} \mathrm{C}$, with final melting for $15 \mathrm{~s}$ at $65^{\circ} \mathrm{C}$. Melting curve analysis was performed to confirm the specificity of the amplification and to identify putative unspecific products. Actin mRNA, set to $100 \%$, was used as an internal standard in all experiments. The quantitative PCR experiments were repeated at least three times for a cDNA prepared from three batches of plants.

\section{Results}

\section{Isolation of egy 1 mutants}

The T-DNA insertion line (SALK-134931, Fig. 1a) (Columbia background) was obtained from the Arabidopsis Biological Resource Center (ABRC) at Ohio State University. The homozygosity of plants was confirmed by PCR amplifying and sequencing. To study the effect of the T-DNA insertion on At5g35220 expression, we analyzed the results of RT-PCR, which showed that this gene was undetectable in the mutant, while it was normal expressed in WT plants (Fig. 1b). The oldest egy1 mutant leaves had the most intense yellow-green phenotype (Fig. 1c). Complementation of egy1 mutants was performed to ensure the function of EGY1 (Fig. 1c).

\section{Yellowing of egy 1 rosette leaves}

The fifth rosette leaves emerged on the same day both in the egy1 mutants and WT plants. The yellow-green of young leaves and albino of old leaves in egy1 mutant (Fig. 2a) was not due to difference in the number of leaves. Yellowing of the egy1 leaves happened first at the tip of the leaf blades, later spread to the end of leaf blades. The leaves sometimes developed chlorosis along the midrib (Fig. 2b-f).

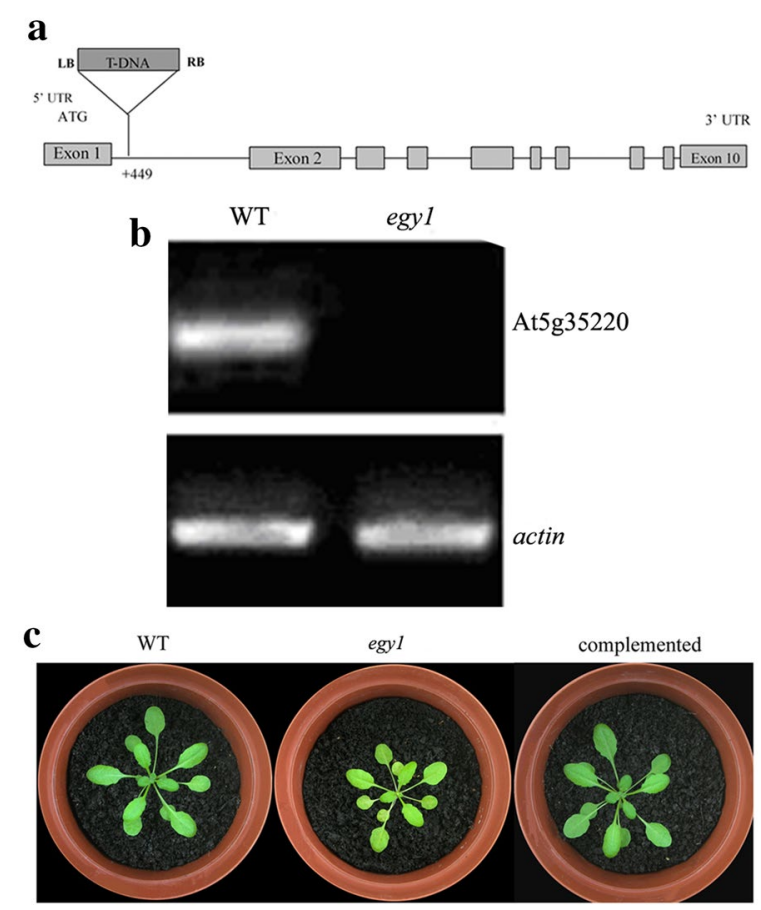

Fig. 1 Expression of EGY1 and phenotypes of egy1 mutants. Gene structure of EGY1 and location of T-DNA insert (a), RT-PCR analysis of EGY1 expression in wild-type and egy 1 leaves $(\mathbf{b})$, phenotypes of wildtype, egy 1 and egy 1 complemented lines at 28 days after germination (c) 


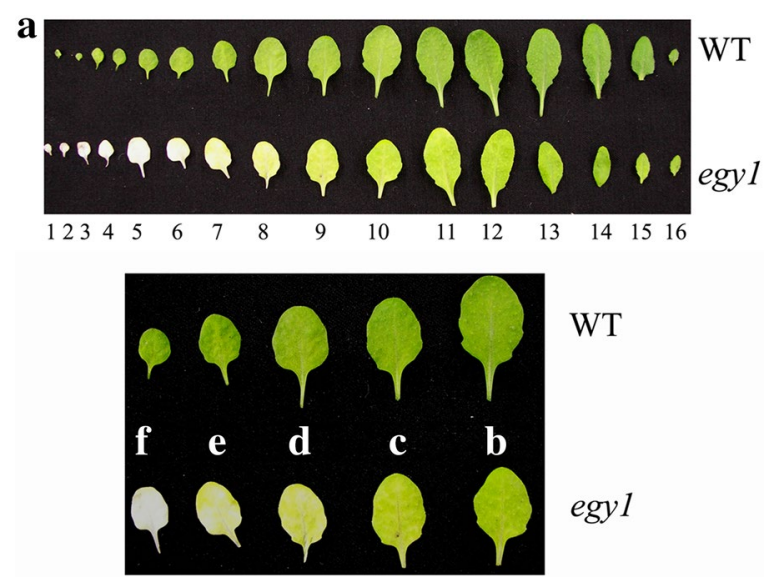

Fig. 2 Phenotypes of egy 1 mutants in age-dependent senescence. A photograph of wild-type and egy 1 plants (a), visible senescence phenotypes in the egy 1 leaves (b-f), leaves were numbered from bottom to top. Chlorosis often initiates from midrib (c), and sometimes chlorotic lesions are formed $(\mathbf{d})$ in egy 1 leaves. Chlorotic area expands from tip to base on an egyl leaf $(\mathbf{e}, \mathbf{f})$

\section{Physiological changes in egy 1 leaves}

In order to examine the physiological state of the egy 1 mutants, the survival, chlorophyll content, $F v / F m$, soluble protein content and ion leakage of the fifth leaves were measured in this study.

We first examined the effect of visual leaf longevity (representing the leaf survival) in the egy 1 mutation (Fig. 3a). We observed that the leaf longevity of egy 1 was shortened by $40.1 \%$. The time it took $50 \%$ of leaf population to survive was 22 days after leaf emergence in the mutants, while it was longer, 37 days, in the WT plants (Fig. 3a).

The chlorophyll content of egy1 mutants at 10 days of leaf emergence was $<50 \%$ of that in the WT plants (Fig. 3b). Chlorophyll content decreased in egy1 mutants after 20 days, while it was stable until 30 days of leaf emergence in WT leaves (Fig. 3b).

The $F v / F m$ ratio, which reflects the photochemical quantum efficiency of PSII, was similar in egy1 mutants and WT plants at 15 days of leaf emergence. But after 20 days, the $F v / F m$ ratio began to decrease in egy 1 leaves, whereas it did not decline until 25 days in WT leaves (Fig. 3c).

At 10 days after leaf emergence, soluble protein contents were measured in egy1 mutants and WT plants. Soluble protein began to decrease on the 15 days of leaf emergence in egy1 mutant, while it increased until 20 days in WT leaves. Protein contents decreased below $60 \%$ of the initial level at 25 days of leaf emergence in egy1 mutants and after 35 days in WT (Fig. 3d).
Ion leakage, an indicator for the intactness of the plasma membrane, began to increase 15 days after leaf emergence in egy 1 leaves, and its ratio continued increasing significantly throughout the growth period. However, ion leakage in WT leaves began to increase until 30 days. Moreover, there was no increase in the ratio throughout the growth period in WT plants, unlike egy1 mutants (Fig. 3e).

\section{Transcription of senescence-related genes in the egy 1 mutants}

To evaluate leaf senescence in the egy1 mutants at molecular level, the transcription levels of SAG12, SAG24, SEN4, $C A B$ and $R B C S$ were examined by RNA gel-blot analysis. The transcripts of $S A G 12$ in egy1 plants accumulated ca. $207 \%$ at 33 days and ca. $400 \%$ at 40 days compared with that of 26 days after germination, which was more than those in WT plants (Fig. 4). The transcripts of SAG24 and SEN4 were also increased in egy1 mutants. Similarly, the transcript levels of $C A B$ and $R B C S$ decreased more obviously in egyl plants than those in WT plants with increasing leaf age (Fig. 4).

\section{The influence of darkness treatment}

27-Day-old WT and egy1 plants (Fig. 5a, b) were placed in a dark chamber in order to observe phenotypic changes due to darkness. After 3 days, the yellow-green phenotype was more obvious in egyl mutants (Fig. 5d) than that in WT plants (Fig. 5c).

\section{Leaf senescence of egy 1 mutants was delayed by exogenously applied glucose}

Cotyledon greening in WT was almost the same as in egy 1 mutants regardless of treatment with or without $1 \%$ (56 mM) and $2 \%(111 \mathrm{mM})$ glucose (Fig. 6a). However, cotyledons greening was inhibited in egy 1 plants treated with $4 \%(222 \mathrm{mM})$ and $6 \%(333 \mathrm{mM})$ glucose, and in WT plants with $6 \%$ glucose (Fig. 6a). Moreover, hypocotyl elongation was increased from 0 to $2 \%$ glucose both in WT and egy1 seedlings (Fig. 6b), while it was inhibited under $4 \%$ and $6 \%$ glucose both in egy1 and WT seedlings (Fig. 6b). Next we planted WT and egy1 mutants on $2 \%$ glucose for 5 weeks. The results showed that yellowgreen phenotype of egy 1 mutants was partially restored by glucose treatment (Fig. 7a). Chlorophyll contents were increased and ion leakage was decreased in egy 1 mutants applied with $2 \%$ glucose compared to those without glucose treatment (Fig. 7b, c).

The results from quantitative PCR analysis indicated that the high expressions of HXK1 and SAG12 in egy1 mutants were impaired by $2 \%$ glucose treatment for 5 weeks (Fig. 8). 

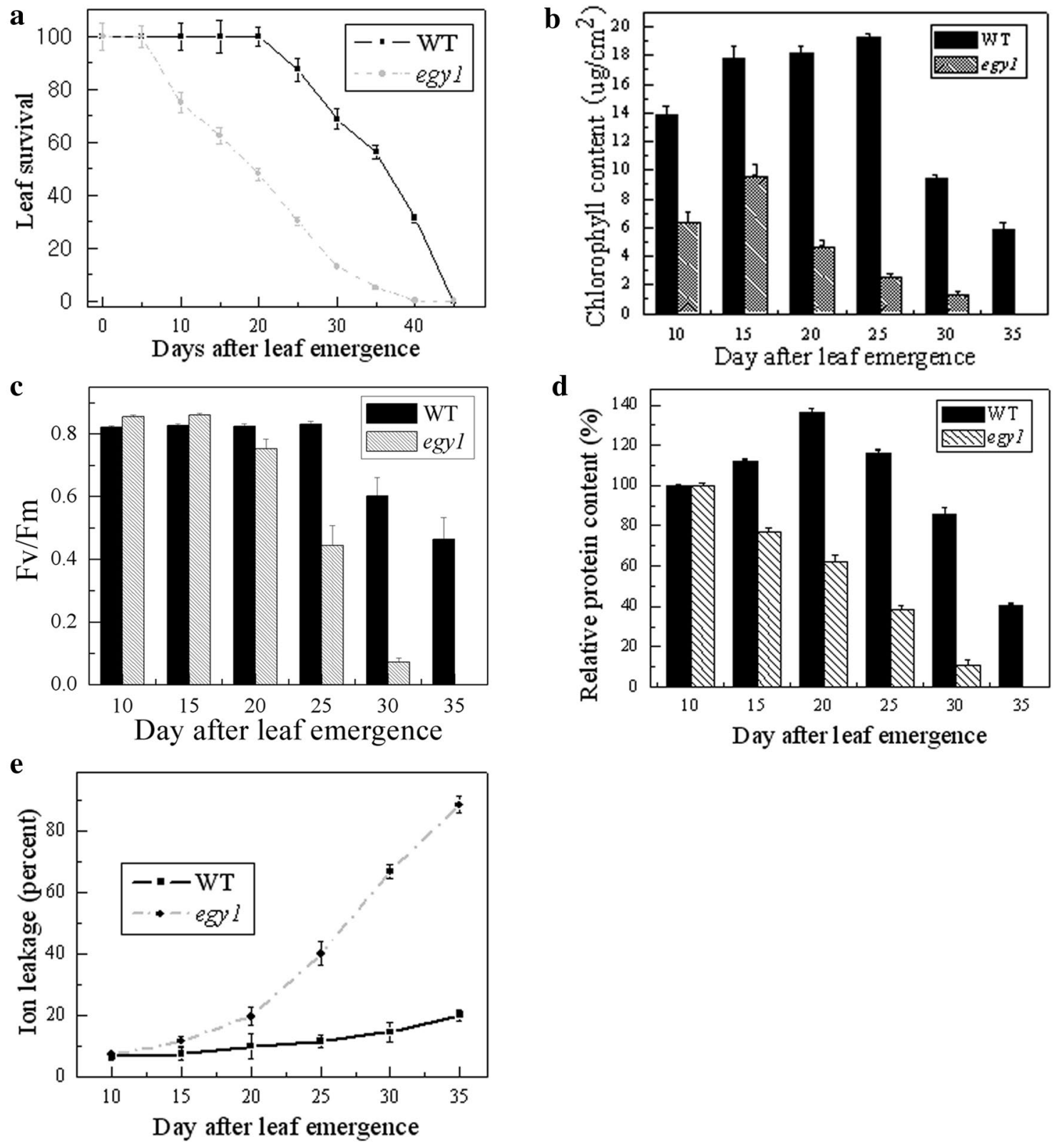

Fig. 3 A time-course of changes in senescence indicators of the fifth leaves at indicated times after leaf emergence. Leaf survival (a), chlorophyll contents per leaf area $(\mathbf{b}), F v / F m(\mathbf{c})$, soluble protein contents per leaf $(\mathbf{d})$ and ion leakage $(\mathbf{e})$. Soluble protein contents were shown as relative values of those measured at 10 days. At 35 days, chlorophyll contents and soluble protein contents were undetected in the egy 1 mutants. The standard deviation was calculated from twelve samples for leaf survival; six for chlorophyll and Fv/Fm, and three for soluble protein and ion leakage measurements

\section{Discussion}

In general, leaf senescence is dependent on age and developmental stage under normal conditions and in the absence of external stress, and the leaf yellowing during senescence is usually observed in old leaves. In this study, the leaf yellowing phenotype of egy1 mutant and WT plants is also related to the increasing leaf age and leaf normal development. However, egy1 mutant leaves are more yellow than those of WT plants at the same age and development stage (Figs. 1, 2), suggesting that egy1 mutants have an early-senescence phenotype.

To further confirm the early-senescence phenotype, we measured the physiological characteristics associated with senescence in egyl mutants. The leaf longevity was 


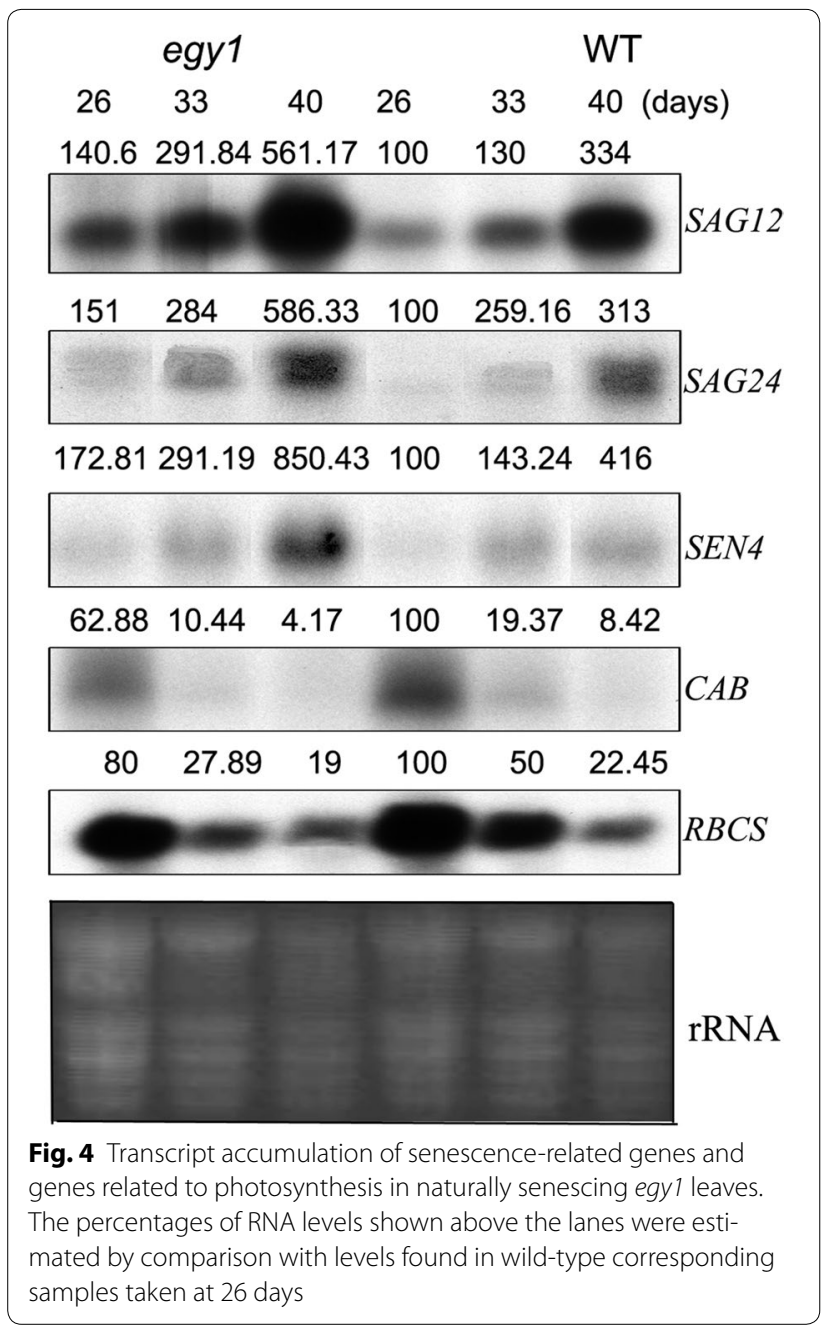

lower in egy1 mutants than that of WT plants (Fig. 3). Similar results were detected in apg7-1 mutants of Arabidopsis which showed an early-senescence phenotype (Doelling et al. 2002). Chlorophyll can provide basic information of photosynthesis and photosynthesis defects is an important feature of senescence. Our results showed that chlorophyll contents were also reduced in egy1 mutants (Fig. 3), which was similar to the reported data by Frick et al. (2003) in porB-1porC-1 double mutant of older seedlings and by Stettler et al. (2009) in mex1 leaves. Proteins are fundamental components of all living cells and are necessary for the proper functioning of an organism, and could be decreased during leaf senescence. We found that egy1 mutation had decreased soluble protein contents (Fig. 3), similar tendency was also detected in hys1 and apg7-1 early-senescence mutants (Doelling et al. 2002; Yoshida et al. 2002). Ion leakage is an indicator of membrane integrity. When leaf senescence, membrane became fragile and leak. In egy1 mutants, ion leakage was

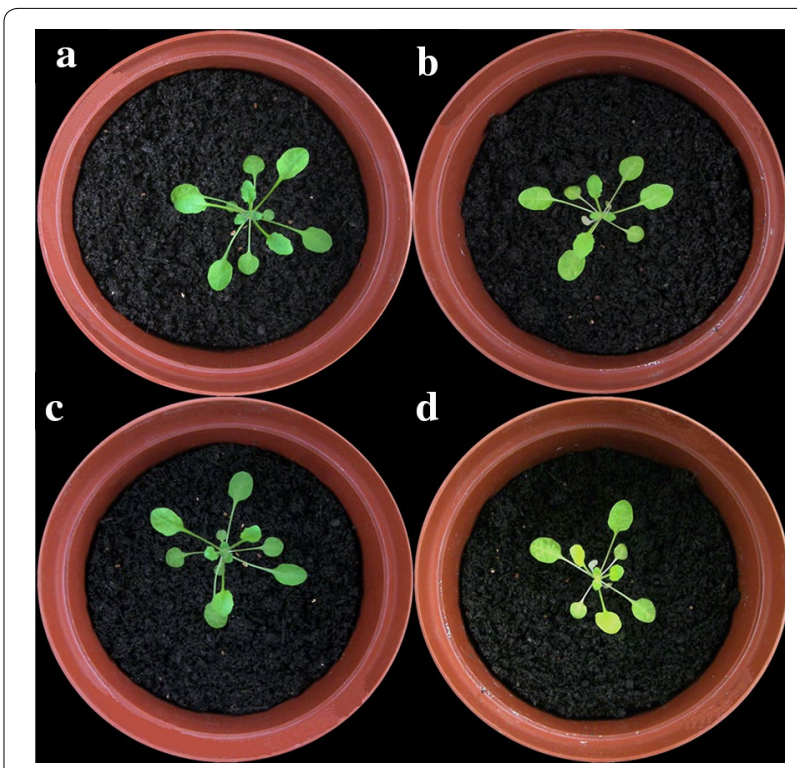

Fig. 5 Yellowing of egy 1 leaves during dark treatment. Wild-type (a, c) and egy $1(\mathbf{b}, \mathbf{d})$ plants before dark treatment $(\mathbf{a}, \mathbf{b})$ and after 3 days (c) d) of dark treatment are shown

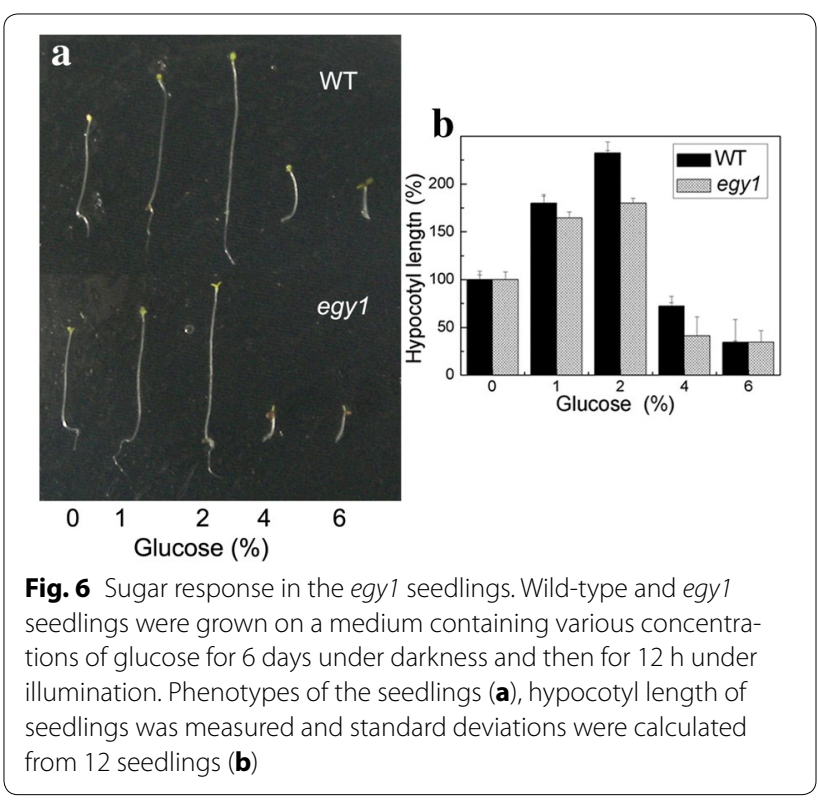

increased (Fig. 3). Taken together, these results suggested that the egy1 mutants had early-senescence traits.

Leaf senescence is accompanied by increased expression of senescence-associated genes (SAGs) and decreased expression of genes related to photosynthesis (Lim et al. 2003; Zentgraf et al. 2004). The transcript levels of SAG12, SAG24 and SEN4 genes were increased in senescent leaves (Wu et al. 2008; Yoshida et al. 2001), while $C A B$ and $R B C S$ genes were down-regulated during leaf 


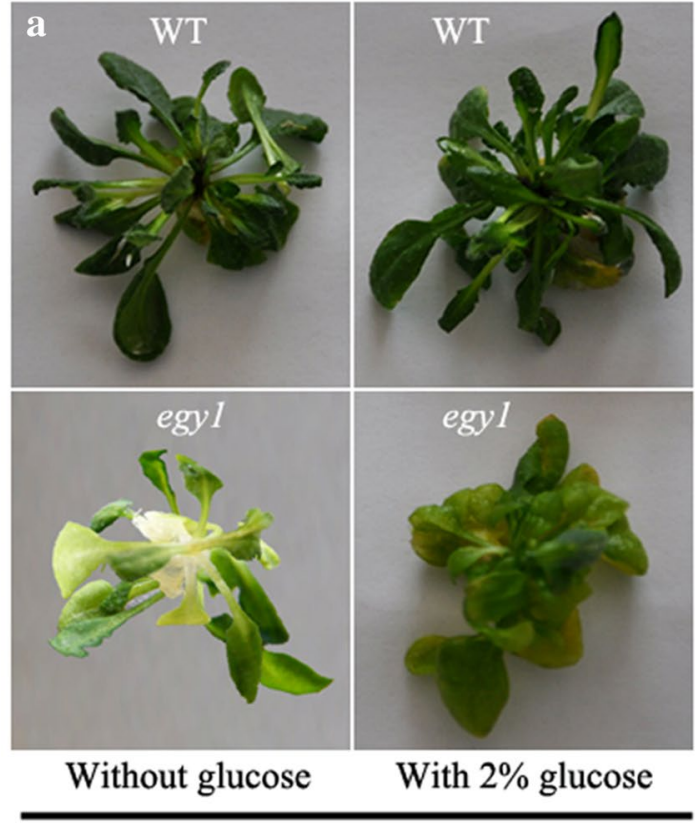

5 weeks
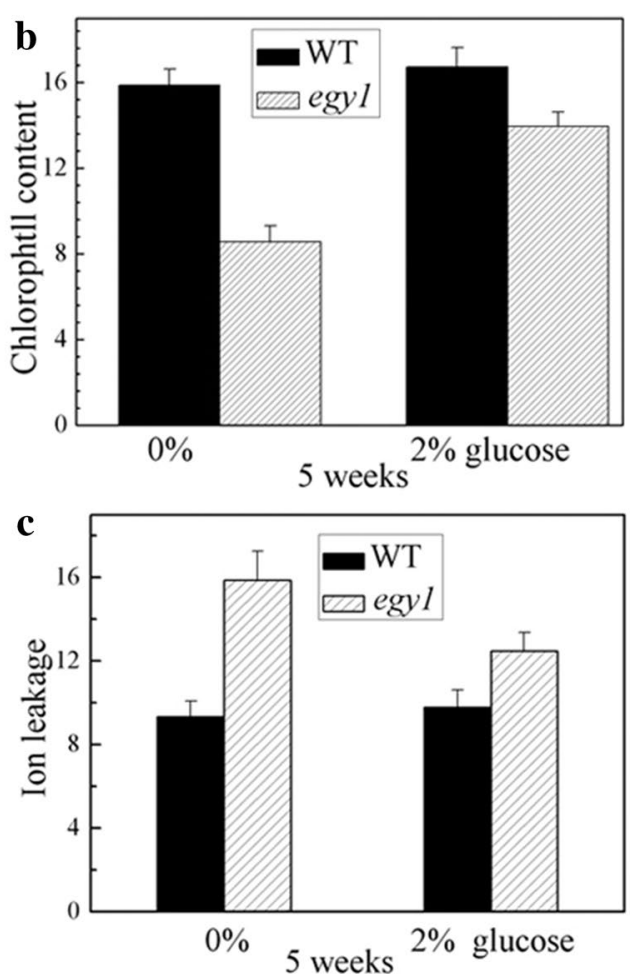

Fig. 7 Phenotypes (a), chlorophyll contents (b) and ion leakage (c) in wild-type and egy 1 plants supplied with $2 \%$ glucose for 5 weeks
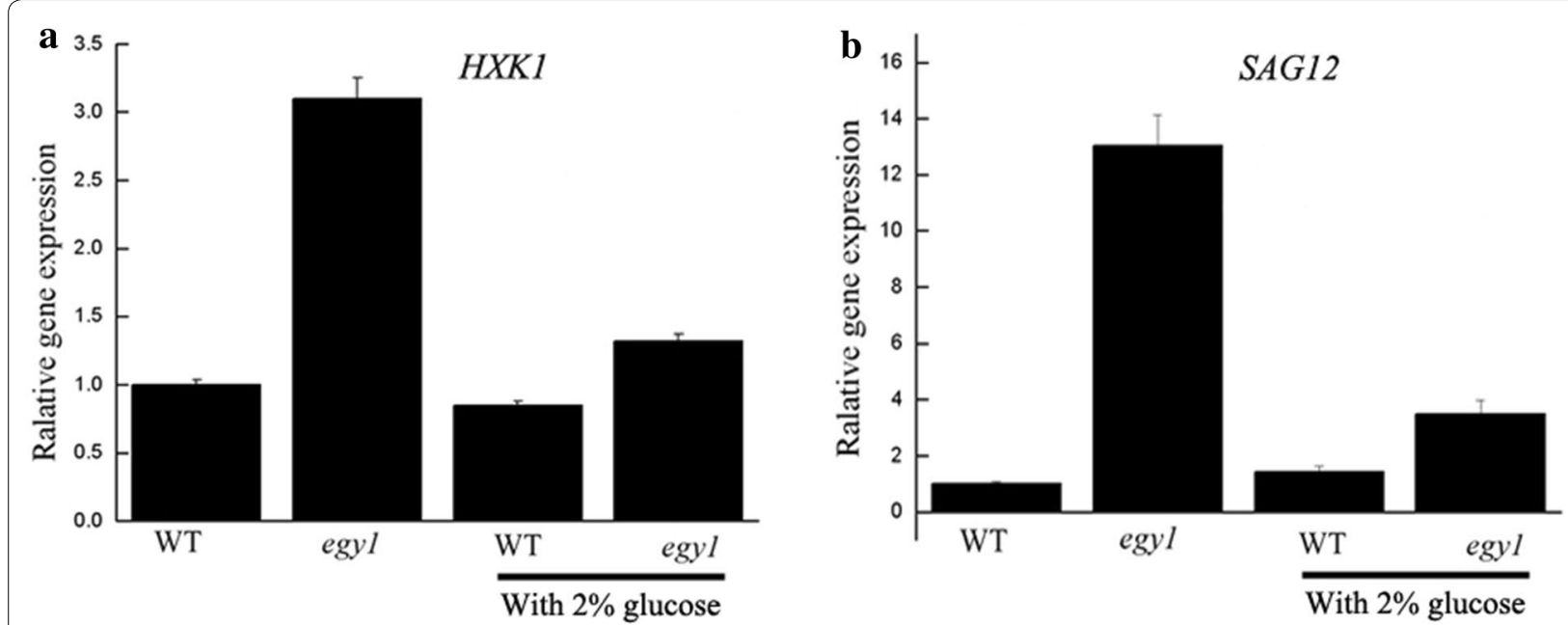

Fig. 8 The relative transcription levels of hexokinase 1 (HXK1) (a) and senescence-associated gene 12 (SAG12) (b) in egy1 and wild-type plants growing on $0 \%$ and $2 \%$ glucose. The transcripts were determined by real-time PCR and normalized against actin. The transcription levels are relative to the WT set at $0 \%$ glucose. The quantitative PCR experiments were repeated at least three times for a cDNA prepared from three batches of plants

senescence (Woo et al. 2001). Similar results were found both in egy1 mutants and in WT plants with increasing leaf age. It is notable that, SAG12, SAG24, SEN4 increased and $C A B$ and $R B C S$ decreased more obviously in egy1 mutants compared to WT plants (Fig. 4). These results indicated that leaf senescence occurred indeed in transcript level in egy1 plants.

Leaf senescence could be induced by external stresses, such as darkness. Darkness treatment induced uniform and rapid senescence (Chrost et al. 2004; del Rio et al. 
2003; Mishev et al. 2009). After 3 days of dark treatment, egy1 mutant leaves were more yellow while the phenotype of WT plants had no obvious changes (Fig. 5), suggesting that egy1 mutants had an early-senescence phenotype under normal conditions and in darkness treatment. So EGY1 should have an important role in leaf normal development in A. thaliana. As the egy1 mutants have a wide variety of senescence symptoms, the egy 1 gene most likely functions as a regulatory component controlling functional leaf senescence rather than as a protein directly executing the senescence process (Kim et al. 2009; Lim et al. 2010).

The chloroplasts in egy1 mutants had reduced granal thylakoids, poorly developed lamellae networks and decreased photosynthetic proteins (Chen et al. 2005). The loss in photosynthesis caused cellular sugar starvation, and lack of energy (Patro et al. 2014). Sugar starvation is known to affect leaf senescence, repress hypocotyl elongation, cotyledon greening and shoot development (Arru et al. 2008; Gazzarrini and McCourt 2001). In tobacco plants, sugar starvation accelerated leaf senescence, suggesting that sugar signaling could directly or indirectly promote leaf senescence (Wingler and Roitsch 2008). This starvation could be restored after exogenously applied sugars through sugar metabolism with complex sugar signaling network. Yoshida et al. (2002) found that hys 1 seedlings with exogenously applied sugars were hyper-responsive to glucose, implying that sugar may play a role in the early induction of senescence. In our study, hypocotyl elongation was increased in egy1 seedlings growing on $2 \%$ glucose. Furthermore, the yellow-green phenotype of egy1 mutants was partially restored by $2 \%$ glucose (Fig. 7), suggesting that the symptom of early senescence was relieved after glucose supplementation. Meanwhile, the expressions of HXK1 and SAG12 (which are usually at a high level in senescence leaves) were obviously decreased in egyl mutants by $2 \%$ glucose (Fig. 8), implying that the early senescence of egy1 mutants may be due to sugar starvation. It therefore could be restored by exogenous glucose treatment. With regard to the inhibition of cotyledons greening or hypocotyl elongation by $4 \%$ or $6 \%$ glucose, it is a result from the effects of high concentrations of glucose in the medium and has been reported in the previous study (Jang et al. 1997).

\section{Conclusion}

In conclusion, our results suggest that leaf senescence induced by EGY1-defection may be due to sugar starvation and can be partially restored by glucose in $A$. thaliana. EGY1 regulated leaf senescence provides key information to understand the molecular mechanism of leave senescence in plants.

\section{Abbreviations}

EGY1: ethylene-dependent gravitropism-deficient and yellow-green 1; SAG: senescence-associated gene; CAB: chlorophyll a/b-binding protein ; RBCS: the rubisco small subunit gene; SEN4: endo-xyloglucan transferase/xyloglucan endo-1,4-beta-D-glucanase.

\section{Authors' contributions}

CYC conceived and designed the experiments. CYC XZ performed the experiments. JW analyzed the data. CYC wrote the paper. All authors read and approved the final manuscript.

\section{Acknowledgements}

This research was supported by National Natural Science Foundation of China (31300226), the National Basic Research Program of China (2013CB429904) and the Natural Science Foundation of Gansu Province (1308RJYA094).

\section{Competing interests}

The authors declare that they have no competing interests.

Received: 30 June 2015 Accepted: 20 January 2016

Published online: 03 February 2016

\section{References}

Aluru MR, Stessman DJ, Spalding MH, Rodermel SR (2007) Alterations in photosynthesis in Arabidopsis lacking IMMUTANS, a chloroplast terminal oxidase. Photosynth Res 91:11-23

Arru L, Rognoni S, Poggi A, Loreti E (2008) Effect of sugars on auxin-mediated LeEXPA2 gene expression. Plant Growth Regul 55:11-20

Balasubramanian R, Karve A, Moore BD (2008) Actin-based cellular framework for glucose signaling by Arabidopsis hexokinase1. Plant Signal Behav 3:322-324

Bellaoui M, Gruissem W (2004) Altered expression of the Arabidopsis ortholog of DCL affects normal plant development. Planta 219:819-826

Buchanan-Wollaston V, Earl S, Harrison E, Mathas E, Navabpour S, Page T, Pink D (2003) The molecular analysis of leaf senescence-a genomics approach. Plant Biotechnol I 1:3-22

Castillo MC, Leon J (2008) Expression of the beta-oxidation gene 3-ketoacylCOA thiolase 2 (KAT2) is required for the timely onset of natural and darkinduced leaf senescence in Arabidopsis. J Exp Bot 59:2171-2179

Chen G, Bi YR, Li N (2005) EGY1 encodes a membrane-associated and ATPindependent metalloprotease that is required for chloroplast development. Plant J 41:364-375

Cho YH, Yoo SD, Sheen J (2006) Regulatory functions of nuclear hexokinase 1 complex in glucose signaling. Cell 127:579-589

Chrost B, Daniel A, Krupinska K (2004) Regulation of alpha-galactosidase gene expression in primary foliage leaves of barley (Hordeum vulgare L.) during dark-induced senescence. Planta 218:886-889

Clough SJ, Bent AF (1998) Floral dip: a simplified method for Agrobacteriummediated transformation of Arabidopsis thaliana. Plant J 16:735-743

del Rio LA, Sandalio LM, Altomare DA, Zilinskas BA (2003) Mitochondrial and peroxisomal manganese superoxide dismutase: differential expression during leaf senescence. J Exp Bot 54:923-933

Doelling JH, Walker JM, Friedman EM, Thompson AR, Vierstra RD (2002) The APG8/12-activating enzyme APG7 is required for proper nutrient recycling and senescence in Arabidopsis thaliana.J Biol Chem 277:33105-33114

Frick G, Su Q, Apel K, Armstrong GA (2003) An Arabidopsis porB porC double mutant lacking light-dependent NADPH:protochlorophyllide oxidoreductases B and C is highly chlorophyll-deficient and developmentally arrested. Plant J 35:141-153

Gazzarrini S, McCourt P (2001) Genetic interactions between ABA, ethylene and sugar signaling pathways. Curr Opin Plant Biol 4:387-391

Guiboileau A, Sormani R, Meyer C, Masclaux-Daubresse C (2010) Senescence and death of plant organs: nutrient recycling and developmental regulation. C R Biol 333:382-391

Guo Y, Cai Z, Gan S (2004) Transcriptome of Arabidopsis leaf senescence. Plant, Cell Environ 27:521-549 
Guo D, Gao X, Li H, Zhang T, Chen G, Huang P, An L, Li N (2008) EGY1 plays a role in regulation of endodermal plastid size and number that are involved in ethylene-dependent gravitropism of light-grown Arabidopsis hypocotyls. Plant Mol Biol 66:345-360

Hortensteiner S, Feller U (2002) Nitrogen metabolism and remobilization during senescence. J Exp Bot 53:927-937

Hung K, Kao C (1998) Involvement of lipid peroxidation in methyl jasmonatepromoted senescence in detached rice leaves. Plant Growth Regul 24:17-21

Jang JC, Leon P, Zhou L, Sheen J (1997) Hexokinase as a sugar sensor in higher plants. Plant Cell 9:5-19

Kakani VG, Reddy KR, Zhao D, Gao W (2004) Senescence and hyperspectral reflectance of cotton leaves exposed to ultraviolet-B radiation and carbon dioxide. Physiol Plant 121:250-257

Kim J-H, Lee MH, Moon YR, Kim J-S, Wi SG, Kim TH, Chung BY (2009) Characterization of metabolic disturbances closely linked to the delayed senescence of Arabidopsis leaves after $\gamma$ irradiation. Environ Exp Bot 67:363-371

Lim PO, Woo HR, Nam HG (2003) Molecular genetics of leaf senescence in Arabidopsis. Trends Plant Sci 8:272-278

Lim PO, Lee IC, Kim J, Kim HJ, Ryu JS, Woo HR, Nam HG (2010) Auxin response factor 2 (ARF2) plays a major role in regulating auxin-mediated leaf longevity. J Exp Bot 61:1419-1430

Loreti E, Povero G, Novi G, Solfanelli C, Alpi A, Perata P (2008) Gibberellins, jasmonate and abscisic acid modulate the sucrose-induced expression of anthocyanin biosynthetic genes in Arabidopsis. New Phytol 179:1004-1016

Mishev K, Stefanov D, Ananieva K, Slavov C, Ananiev E (2009) Different effects of dark treatment on pigment composition and photosystem I and II activities in intact cotyledons and primary leaves of Cucurbita pepo (zucchini). Plant Growth Regul 58:61-71

Ono K, Nishi Y, Watanabe A, Terashima I (2001) Possible mechanisms of adaptive leaf senescence. Plant Biol 3:234-243

Patro L, Mohapatra PK, Biswal UC, Biswal B (2014) Dehydration induced loss of photosynthesis in Arabidopsis leaves during senescence is accompanied by the reversible enhancement in the activity of cell wall $\beta$-glucosidase. J Photochem Photobiol, B 137:49-54

Sambrook J, Russell DW (2001) Molecular cloning: a laboratory manual, 3rd edn. Cold Spring Harbor Laboratory Press, New York

Sims DA, Gamon JA (2002) Relationships between leaf pigment content and spectral reflectance across a wide range of species, leaf structures and developmental stages. Remote Sens Environ 81:337-354
Smeekens S, Ma J, Hanson J, Rolland F (2009) Sugar signals and molecular networks controlling plant growth. Curr Opin Plant Biol 13:274-279

Stettler M, Eicke S, Mettler T, Messerli G, Hortensteiner S, Zeeman SC (2009) Blocking the metabolism of starch breakdown products in Arabidopsis leaves triggers chloroplast degradation. Mol Plant 2:1233-1246

van Doorn WG (2008) Is the onset of senescence in leaf cells of intact plants due to low or high sugar levels? J Exp Bot 59:1963-1972

Vinti G, Fourrier N, Bowyer JR, Lopez-Juez E (2005) Arabidopsis cue mutants with defective plastids are impaired primarily in the photocontrol of expression of photosynthesis-associated nuclear genes. Plant Mol Biol 57:343-357

Wiedemuth K, Muller J, Kahlau A, Amme S, Mock HP, Grzam A, Hell R, Egle K, Beschow H, Humbeck K (2005) Successive maturation and senescence of individual leaves during barley whole plant ontogeny reveals temporal and spatial regulation of photosynthetic function in conjunction with C and N metabolism. J Plant Physiol 162:1226-1236

Wingler A, Roitsch T (2008) Metabolic regulation of leaf senescence: interactions of sugar signalling with biotic and abiotic stress responses. Plant Biol (Stuttg) 10(Suppl 1):50-62

Woo HR, Chung KM, Park JH, Oh SA, Ahn T, Hong SH, Jang SK, Nam HG (2001) ORE9, an F-box protein that regulates leaf senescence in Arabidopsis. Plant Cell 13:1779-1790

Wu K, Zhang L, Zhou C, Yu CW, Chaikam V (2008) HDA6 is required for jasmonate response, senescence and flowering in Arabidopsis. J Exp Bot 59:225-234

Xiao W, Sheen J, Jang JC (2000) The role of hexokinase in plant sugar signal transduction and growth and development. Plant Mol Biol 44:451-461

Yoshida S, Ito M, Nishida I, Watanabe A (2001) Isolation and RNA gel blot analysis of genes that could serve as potential molecular markers for leaf senescence in Arabidopsis thaliana. Plant Cell Physiol 42:170-178

Yoshida S, Ito M, Nishida I, Watanabe A (2002) Identification of a novel gene HYS1/CPR5 that has a repressive role in the induction of leaf senescence and pathogen-defence responses in Arabidopsis thaliana. Plant J 29:427-437

Zentgraf U, Jobst J, Kolb D, Rentsch D (2004) Senescence-related gene expression profiles of rosette leaves of Arabidopsis thaliana: leaf age versus plant age. Plant Biol (Stuttg) 6:178-183

\section{Submit your manuscript to a SpringerOpen ${ }^{\circ}$ journal and benefit from:}

- Convenient online submission

- Rigorous peer review

- Immediate publication on acceptance

- Open access: articles freely available online

- High visibility within the field

- Retaining the copyright to your article

Submit your next manuscript at $>$ springeropen.com 